\title{
SECOND HARMONIC GENERATION IN SPIN-GLASS MICROSTRUCTURES AND FABRICATION OF MICROSTRUCTURES IN IV-VI EPILAYERS*
}

G. Grabecki, T. Dietl, Marek Cieplak, W. Plesiewicz, A. Lenard, T. SKośKiewicz

Institute of Physics, Polish Academy of Sciences

Al. Lotników 32/46, 02-668 Warszawa, Poland

E. Kamińska, A. Piotrowska, R. Żarecka

Institute of Electron Technology, Al. Lotników 32/46, 02-668 Warszawa, Poland

\section{G. SPRINGHOLZ AND G. BaUer}

Institut für Halbleiterphysik, Johannes Kepler Universität Linz, 4040 Linz, Austria

We studied magnetoconductance of two different mesoscopic systems: microregions containing two-dimensional electron gas adjacent to a grain-boundary plane in bicrystals of $\mathrm{Hg}_{0.79} \mathrm{Cd}_{0.19} \mathrm{Mn}_{0.02} \mathrm{Te}$ and photolithographically patterned microstructures of $\mathrm{Pb}_{1-x} \mathrm{Mn}_{x} \mathrm{Se}$. In both systems universal conductance fluctuations and generation of the second-harmonic voltage were observed at $T \leq 1 \mathrm{~K}$. Moreover, in the former system the second harmonic signal exhibited distinct rise when the sample was cooled below the spin-glass freezing temperature $(100 \mathrm{mK})$.

PACS numbers: 71.55.Jv

Research on quantum transport in semiconductors has progressed from bulk materials and layered structures to patterned devices of much greater complexity and novel properties. Microstructures that incorporate diluted magnetic semiconductors are particularly interesting in this context as they may exhibit new effects driven by spin-exchange interactions. It has been widely believed that studies of mesoscopic transport phenomena in magnetic materials offer a worthwhile opportunity to probe dynamics of the spin subsystem [1].

We undertook magnetoconductance studies of two different types of mesoscopic systems based on two different semimagnetic semiconductors:

*This work was supported by a grant no. 710/2/91 from the Committee for Scientific Research. 
1. Micro four-probe contacts connected to the two-dimensional inversion layers adjacent to a grain-boundary plane in bicrystals of $p-\mathrm{Hg}_{1-x-y} \mathrm{Cd}_{y} \mathrm{Mn}_{x} \mathrm{Te}$ [2]. For the studied Mn concentration, $x=2 \%$, according to our a.c. magnetic susceptibility measurements, the spin-glass freezing occurs at $100 \mathrm{mK}$.

2. Microstructures of lead chalcogenides with manganese and europium in the form of two-terminal wires and six-terminal Hall bars, with the line width $2 \div 4 \mu \mathrm{m}$ (see Fig. 1). We elaborated a fabrication process of such structures from thin films (thickness $D=2 \div 5 \mu \mathrm{m}$ ) of $\mathrm{Pb}_{1-x} \mathrm{Mn}_{x} \mathrm{Se}$ and $\mathrm{Pb}_{1-x} \mathrm{Eu}_{x} \mathrm{Te}$, grown by molecular beam epitaxy (MBE) on a $\mathrm{BaF}_{2}$ substrate. To avoid thermal degradation of the material some modifications of conventional photolithography were introduced. $\mathrm{An}^{\mathrm{SiO}_{2}}$ etch-mask of a thickness of $0.3 \mu \mathrm{m}$ was deposited by RF magnetron sputtering and patterned by photolithography. Finally, the microstructures were formed by Ar ion milling in RF sputtering system under carefully adjusted experimental conditions.
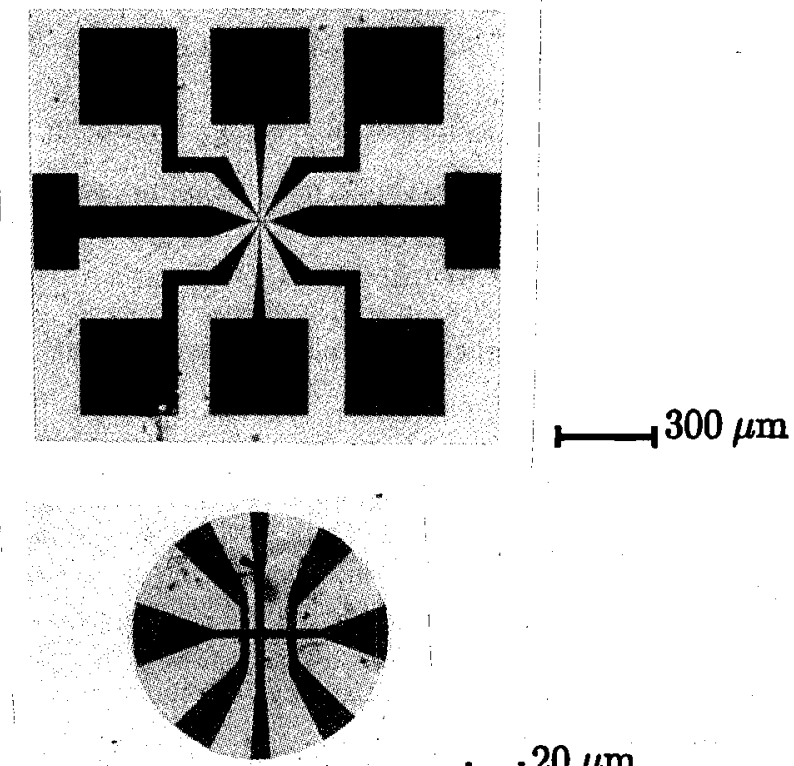

$\longmapsto 20 \mu \mathrm{m}$

Fig. 1. General view of the $\mathrm{Pb}_{1-x} \mathrm{Mn}_{x}$ Se photolithographically patterned sample containing the Hall bridge with the arms width of $2 \mu \mathrm{m}$ (shown below in magnified scale).

Transport properties of the above systems were examined in the $\mathrm{He}^{3} / \mathrm{He}^{4} \mathrm{di}-$ lution refrigerator capable of producing temperatures down to $20 \mathrm{mK}$. In $\mathrm{HgCdMnTe}$, in addition to the universal conductance fluctuations (UCF), discussed in detail elsewhere [3], we found strongly nonlinear current-voltage characteristics, detected via the presence of a signal at the second harmonic of the frequency of the excitation current (typically $109 \mathrm{~Hz}$ ). As shown in inset to Fig. 2, this signal exhibits aperiodic fluctuations as a function of the magnetic field but to 


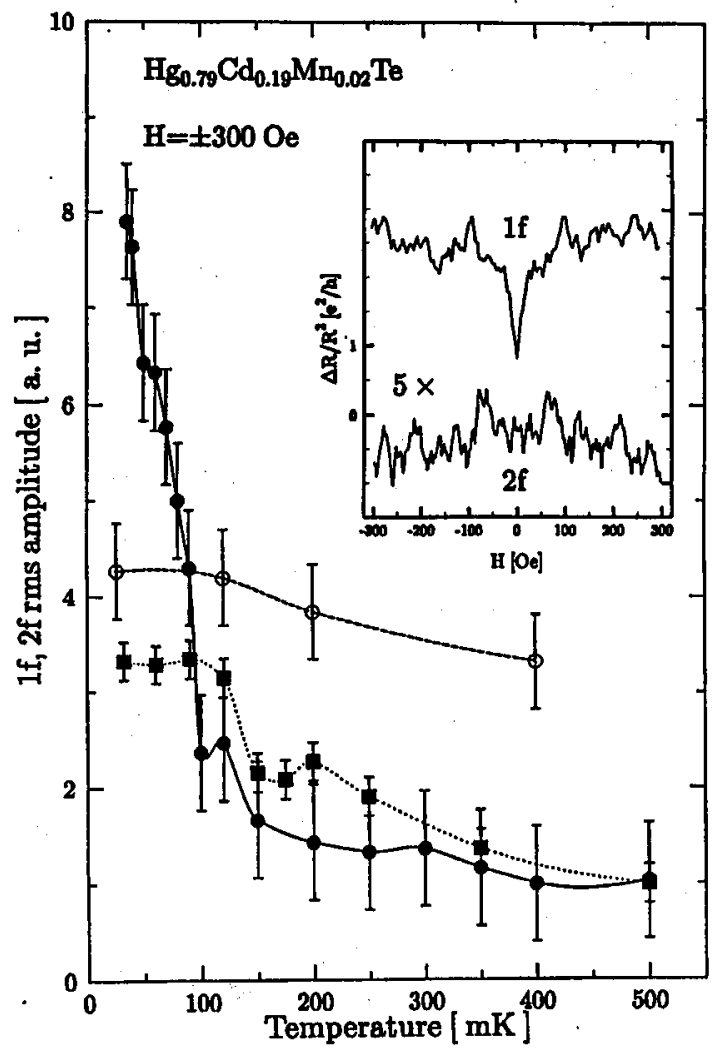

Fig. 2. Temperature dependence of the fluctuation amplitude at the second harmonic in the $\mathrm{HgCdMnTe}$ microstructure for two measurements separated by heating to $300 \mathrm{~K}$ (full circles and squares). The open symbols show data at the fundamental frequency. Inset shows voltage changes (normalized to the current amplitude) as a function of the magnetic field for the same sample at the fundamental and second harmonic of the excitation current (100 and $300 \mathrm{nA}$, respectively; sample resistance $R=99.6 \Omega$ ).

a large extent is symmetric around $H=0$, a property important when assessing the microscopic origin of second harmonic generation [4]. Similarly to the case of GaAs/AlGaAs microstructures [5], its magnitude shows first a square and then linear dependence on the current, varied in our measurements from $30 \mathrm{nA}$ to $1 \mu \mathrm{A}$. Furthermore, in contrast to the signal at the fundamental frequency the magnitude of the signal at the second harmonics changes strongly below $150 \mathrm{mK}$, as depicted in Fig. 2. In spite of some discrepancies between two independent measurements (caused probably by external high-frequency noise) we assign the low-temperature increase in the signal to the spin-glass freezing. In particular, we take our data as an experimental indication for the dependence of the current-voltage characteristics upon the inelastic processes. In order to elucidate the microscopic origin 
of the nonlinear effects, we started numerical calculations of the conductance in -microstructures under the presence of a constant electric field. The results accumulated so far confirm an unusually strong sensitivity of mesoscopic objects to such a perturbation, as well as reproduce a linear dependence of the average second harmonic voltage on the current amplitude.

Classical transport measurements performed on the $\mathrm{Pb}_{1-x} \mathrm{Mn}_{x}$ Se micro Hall bridges at $T=4.2 \mathrm{~K}$ showed that the electron concentration $n_{\mathrm{H}}=1 \pm 0.2 \times 10^{17} \mathrm{~cm}^{-3}$ and mobility $\mu_{\mathrm{H}}=6 \pm 1 \times 10^{4} \mathrm{~cm}^{2} /(\mathrm{V} \mathrm{s})$ values are nearly the same as those measured in the reference macroscopic samples (with size $>100 \mu \mathrm{m}$ ). Thus, the microstructurization process does not degrade the quality of the material. As the temperature was lowered down to millikelvin range, $\mathrm{Pb}_{1-x} \mathrm{Mn}_{x} \mathrm{Se}$ microstructures also showed the presence of reproducible conductance fluctuations with the characteristic amplitude $e^{2} / h$, as well as second harmonic generation. Further studies of these interesting effects are under way.

\section{References}

[1] S. Feng, A.J. Bray, P.A. Lee, M.A. Moore, Phys. Rev. B 36, 5624 (1987).

[2] G. Grabecki, A. Wittlin, T. Dietl, P.A.A. Tennissen, S.A.J. Wiegers, J.A.A.J. Perenboom, Semicond. Sci. Technol 8, S95 (1993).

[3] G. Grabecki, J. Jaroszyński, A. Lenard, W. Plesiewicz, T. Skośkiewicz, T. Dietl, E. Kamińska, A. Piotrowska, G. Springholz, G. Bauer, Proc. 21st Int. Conf. on the Physics of Semiconductors, Beijing 1992, in press; T. Dietl, G. Grabecki, J. Jaroszyński, Semicond. Sci. Technol. 8, S141 (1993).

[4] P.G.N. de Vegvar, G. Timp, P.M. Mankiewich, J.E. Cunningham, R. Behringer, R.G. Howard, Phys. Rev. B 38, 4326 (1988); see also S. Washburn, R.A. Webb, Rep. Prog. Phys. 55, 1311 (1992).

[5] P.G.N. de Vegvar, Phys. Rev. Lett. 70, 837 (1993). 